\title{
EVALUACIÓN DE HÍBRIDOS DE MAÍZ (Zea mays L.) DE GRANO BLANCO Y AMARILLO EN AMBIENTES DE CENTROAMÉRICA, PANAMÁ Y EL CARIBE EN 1996
}

\author{
Adán Aguiluz ${ }^{2}$
}

\begin{abstract}
RESUMEN
Evaluación de híbridos de maíz (Zea mays L.) de grano blanco y amarillo en ambientes de Centroamérica, Panamá y el Caribe en 1996. En 1996, se evaluaron 30 híbridos de grano blanco en 12 localidades, y 20 híbridos de grano amarillo en 14 localidades. Se empleó el testigo HB-83 para grano blanco y el testigo HB-46 para el grano amarillo. La medida de la interacción genotipo-ambiente se obtuvo por el análisis de efectos principales aditivos e interacciones multiplicativas (modelo AMMI). Los híbridos blancos A-7573, H53, HN-951, A-7530, CB-XHS-7GMl y CML-9xCML-47 superaron o igualaron al testigo $\mathrm{HB}-83$ en 17,$8 ; 0,1 ; 1,1 ; 9,2$; 8,1 y $18,7 \%$, respectivamente mostrando poca interacción con el ambiente (valores AMMI cercanos a O). Dos de estos híbrido $\mathrm{s}$ pertenecen a los programas nacionales y tres a las compañías privadas de semilla. Para los híbridos amarillos, sólo los híbridos, CB-XHS-8GM3, HS-6 y DK-888A, superaron al testigo HA-46 en rendimiento y mostraron puntuaciones AMMI cercanas a cero $(0,20 ;-0,07$ y $-0,12$, respectivamente), todos pertenecen a compañías privadas de semilla.
\end{abstract}

\begin{abstract}
EvaIuation of white and yellow grain corn hybrids (Zea mays L.) in environments of Central America, Panama and the Caribbean in 1996. Thirty white-grain hybrids in 1210cations and 20 yellow-grain hybrids in 14 locations were evaluated in 1996. The control HB-83 was used for white grains, and control HB-46 was used for yellow grains. Measurement of genotype/environment interaction was obtained through the analysis of additive main effects and multiplicative in eractions (AMMI model). White hybrids A7573, H-53, HN-951, A-7530, CB- XHS-7GMl and CML9XCML-47 equaled or surpassed the HB-83 control in 17,8\%; $0,1 \% ; 1,1 \% ; 9,2 \% ; 8,1 \%$ and $18,7 \%$ ofthe cases, respectively, showing little interaction with the environment (AMMI values close to O). Two of these hybrids are from national programs, and three from private seed companies. As for the yellow grains, only the CB-XHS-8GM3, HS-6 and DK-888A hybrids surpassed the control HA-46 in yield, and showed AMMI scores c10se to O: $(0,20 ;-0,07$ and $-0,12$, respectively); all of these belong to private seed companies.
\end{abstract}

\section{INTRODUCCION}

El maíz es el cultivo de mayor importancia en la región de Centro América y el Caribe de acuerdo al área de producción y consumo humano y elaboración de concentrados para la alimentación animal. Este cultivo se produce en una gran diversidad de ambientes contrastantes, muchas veces con baja productividad. A pesar de más de tres décadas de investigación desarrollados por los Programas Nacionales de la Región en el desarrollo de germoplasma mejorado, todavía se obtienen muy bajos rendimientos consecuencia de varios factores como son: climáticos (mala distribución de las lluvias), poca fertilidad de suelos, ya que un alto por- centaje del área sembrada se realiza en las laderas, además se utiliza un manejo agronómico inadecuado en ciertas ocasiones no se aprovecha o no se dispone del material genético más adecuado en la región.

Debido a las limitaciones que se tienen en la disponibilidad de cultivares con alto potencial de rendimiento y con buena estabilidad, el Programa Regional de Maíz (PRM), con la colaboración de las compañías privadas desarrolla todos los años y promueven los híbridos nuevos a través de los ensayos uniformes del PCCMCA, ya que es un mecanismo apropiado para que tanto los Programas Nacionales como las compañías privadas puedan poner a disposición, su semilla, en manos de los productores de maíz.

\footnotetext{
1 Presentado en la XLIII Reunión Anual del PCCMCA, Panamá. 1997.
}

2 Coordinador del Programa de Granos Básicos, CENTA, El Salvador. 
El objetivo de este trabajo fue obtener información del comportamiento agronómico de híbridos blancos y amarillos provenientes de los Programas Nacionales y las Empresas Privadas de Semillas a través de diversos ambientes de la región, para identi-ficar los materiales superiores.

Los fitomejoradores frecuentemente enfrentan un problema de gran magnitud cuando seleccionan en presencia del fenómeno de interacción genotipo ambiente (GxA). Para evitar esta interferencia se han diseñado modelos de estabilidad que contribuyen a disminuir el riesgo involucrado en la selección al realizar estimaciones empíricas imperfectas (Córdova 1989). Crossa; Gauch; Zobel (1988), menciona que el modelo AMMI o también llamado Biplot, combina análisis de varianza (ANOVA) y análisis por coordenadas principales (PCA) en una forma integrada. El modelo AMMI ajusta primero los factores aditivos de genotipo $(\mathrm{G})$ y ambiente (A) usando ANOVA y los residuales, que es la interacción GxA lo ajusta a través de PCA. El mismo autor encontró que AMMI 1 basado en dos repeticiones es tan preciso en predicción como la media de tratamiento estimada con 8,61 repeticiones.

Los híbridos blancos HB-85, CB-XHS-507, H-53 y MAX-397 con rendimiento de 6.276, 6.196, 6.061 y $6.012 \mathrm{~kg} /$ ha y puntuaciónAMMI $-0,261 ; 0,022 ; 0,039 \mathrm{y}$ 0,002 ; respectivamente, fueron los más estables del ensayo del PCCMCAde 1991, a la vez superaron el testigo HB-83M $(5.418 \mathrm{~kg} / \mathrm{ha}$, valor $\mathrm{AMMI}=0,136)$ y a la media general (Quemé; Fuentes 1992). Estos autores identificaron por medio del Modelo AMMI, los híbridos amarillos con menos interacción, éstos fueron el EXP-9122, 3078, HC-78 y H-I04, los cuales superaron en rendimiento de grano al testigo HA-45 en 22, 15 y $14 \%$ respectivamente.

Mediante el análisis AMMI, Urbina (1993) determinó que los ruoridos blancos que menos interactuaron con el ambiente (Valores AMMI cercanos a $\mathrm{O}$ ) fueron H-53, HS-5GM; 1402 CW, CB-XHS-511 y HN-913 (0,$07 ;-0,06 ; 0,07 ; 0,09$ y 0,08 valores AMMI) superando en rendimiento con 9,12 y $16 \%$ al testigo HB-83. Este autor indicó que la estabilidad fue menor en los híbridos amarillos que en los blancos ya que sólo el HA-55 y T-9 presentaron puntuaciones AMMI cercanos a cero $(-0,07$ y 0,09$)$, sin embargo sus rendimientos fueron inferiores al promedio global de los ruoridos evaluados. Los híbridos amarillos XL-380 y HR-12 rindieron 35 y $41 \%$ más que el testigo HA-46 y la magnitud de su interacción con el ambiente fue relativamente baja (-0.11 y -0.12 de AMMI), por lo que aparecen promisorios.

Alvarado; Camargo; Gordón (1994) señalan que los mejores híbridos amarillos fueron el EXP-9122 y
CUB- T-4, los cuales superaron en rendimiento al testigo HA-46 (3,83 t/ha) en 19 y $10 \%$, respectivamente, con valores AMMI cercanos a cero, lo cual los identifica como cultivares con baja interacción con el ambiente. Los mejores híbrido s blancos fueron HS-7GM y HB30, que superaron en rendimiento al testigo HB-83 $(4,97 \mathrm{t} / \mathrm{ha})$ en un rango comprendido entre 2 y $3 \%$ y con valores AMMI cercanos a cero. Los mejores híbridos amarillos y blancos además de tener buen rendimiento y poca interacción con el ambiente presentaron adecuadas características agronómicas. Pixley (1995). En la evaluación de los híbridos blancos encontró que los híbridos de mayor estabilidad fueron SEMESA-474, SEMESA-481, XB-I112 y XB-1114, con puntajes AMMI cercanos a cero $(-0,047 ;-0,052 ;-0,003$ y $-0,017$; respectivamen-te), pero no superaron en rendimiento al testigo HB-85 (4,51 t/ha). En cuanto a rendimiento los mejores híbridos fueron $\mathrm{H}-53$ en 5,32 t/ha y $3001 \mathrm{~W}$ en 5,60 t/ha. Entre los híbridos amarillos los de mayor estabilidad fueron XA-I008 y el 3018 con puntajes AMMI cercanos a cero (-0,033 y 0,055; respectivamente).

Brizuela (1996), en la evaluación regional del PCCMCA en 1995, encontró que los híbridos Pioneer 1394BN y CBHS-7GMl fueron superiores al testigo HB-83 (4,76 t/ha) en 4 y 3,5\%, respectivamente con puntuaciones AMMI cercanas a cero. El comportamiento de los moridos blancos fue similar en las localidades de San Jerónimo, Cuyuta y EAP, ya que no se encontró significancia estadística. En los materiales amarillos no hubo respuesta en Lepaguare, Cuyuta y La Máquina. Sin embargo, el análisis AMMI encontró alta significancia en todas las fuentes de variación. Los híbridos amarillos más estables fueron HE-9126, P-8916, P-8812 y DK-999 con rendimientos superiores al testigo (HA-46 con 3,75 t/ha) en 17,$8 ; 18,6 ; 22,6$ y 27,2\%, respectivamente. El híbrido DK-888 superó al testigo en $38,14 \%$.

\section{MATERIALES Y METODOS}

\section{Material genético}

Los ensayos uniformes del PCCMCA de 1996 constaron de 30 híbrido s de grano blanco y 20 amarillos generados por los programas nacionales y compañías privadas productoras de semilla. La descripción de las introducciones se presenta en los Cuadros 1 y 2 .

\section{Número de ensayos y localidades de evaluación}

Se evaluaron 12 ensayos de híbridos de grano blanco y 14 de grano amarillo, los cuales se distribuyen en 
Cuadro 1. Ensayo de híbridos de maíz de grano blanco del PCCMCA. 1996.

\begin{tabular}{|c|c|c|c|}
\hline Entrada & Híbrido & Institución/Empresa & País \\
\hline 1 & HB-83 & ICTA & Guatemala \\
\hline 2 & HB-85 & ICTA & Guatemala \\
\hline 3 & HE-9101 & ICTA & Guatemala \\
\hline 4 & HN-955A & INTA & Nicaragua \\
\hline 5 & HN-953 & INTA & Nicaragua \\
\hline 6 & HN-951 & INTA & Nicaragua \\
\hline 7 & MBHB-51 & DICTA & Honduras \\
\hline 8 & MBHB-52 & DICTA & Honduras \\
\hline 9 & $\mathrm{H}-53$ & CENTA & El Salvador \\
\hline 10 & HE-59 & CENTA & El Salvador \\
\hline 11 & $\mathrm{C}-220$ & CARGIL & México \\
\hline 12 & C-526 & CARGIL & México \\
\hline 13 & C-381 & CARGIL & México \\
\hline 14 & AGX-5488 & AGROCERES & Brasil \\
\hline 15 & DK-880 & DEKALB & U.S.A. \\
\hline 16 & CB-XHS-5GM1 & CRISTIANI BURKARD & Guatemala \\
\hline 17 & CB-XHS-5GM2 & CRISTIANI BURKARD & Guatemala \\
\hline 18 & CB-XHS-7GM1 & CRISTIANI BURKARD & Guatemala \\
\hline 19 & A-775 & ASGROW & México \\
\hline 20 & A-7530 & ASGROW & México \\
\hline 21 & A-XM-7598 & ASGROW & México \\
\hline 22 & A-7573 & ASGROW & México \\
\hline 23 & XM-7595 & ASGROW & México \\
\hline 24 & MAX-325 & AGRIDEC & México \\
\hline 25 & MAX-329 & AGRIDEC & México \\
\hline 26 & MAX-327 & AGRIDEC & México \\
\hline 27 & HR-93M & SEMINAL & Guatemala \\
\hline 28 & HR-93 & SEMINAL & Guatemala \\
\hline 29 & CML-247xCML-254 & PRM-CIMMYT & México \\
\hline 30 & CML-9xCML-47 & PRM-CIMMYT & México \\
\hline
\end{tabular}

Cuadro 2. Ensayo de híbridos de grano amarillo del PCCMCA. 1996.

\begin{tabular}{rlll}
\hline Entrada & \multicolumn{1}{c}{ Híbrido } & Institución/Empresa & \multicolumn{1}{c}{ País } \\
\hline 1 & RD-662 & CESDA & Rep. Dominicana \\
2 & MBHA-53 & DICTA & Honduras \\
3 & MBHA-54 & DICTA & Honduras \\
4 & C-805 & CARGIL & México \\
5 & C-701 & CARGIL & México \\
6 & C-425 & CARGIL & México \\
7 & HA-46 & ICTA & Guatemala \\
8 & HE-9122 & ICTA & Guatemala \\
9 & HE-9126 & ICTA & Guatemala \\
10 & H-104 & CENTA & El Salvador \\
11 & P-8916 & IDIAP & Panamá \\
12 & P-8812 & IDIAP & Panamá \\
13 & DK-888A & DEKALB & USA \\
14 & AG-51O & AGROCERES & Brasil \\
15 & AG-9014 & AGROCERES & Brasil \\
16 & CB-XHS-8GM2 & CRISTIANI BURKARD & Guatemala \\
17 & CB-XHS-8GM3 & CRISTIANI BURKARD & Guatemala \\
18 & HS-6 & CRISTIANI BURKARD & Guatemala \\
19 & CML-287xCL-331 & PRM - CIMMYT & México \\
20 & ESA4xESA2 & CENTA & El Salvador \\
\hline
\end{tabular}


toda la región de Centro América, El Caribe, Panamá, México y Brasil. Las localidades en donde se establecieron los ensayos se presentan en los Cuadros 3 y 4 .

\section{Diseño experimental}

En el ensayo uniforme de hfbridos blancos se utilizó un diseño de látice rectangular 6 x 5 con tres repeticiones y cuatro surcos de 5,5 $\mathrm{m}$ de largo por unidad ex- perimental. En el ensayo de hfbridos amarillos, se utilizó un diseño de látice rectangular 5 x 4 con tres repeticiones y el número de surcos fue de cuatro, igual que en los híbridos blancos. El número de plantas por surco de 5,5 m de largo fue de 22 considerándose los dos surcos centrales como la parcela útil con área de $8,8 \mathrm{~m}^{2}$. El testigo del ensayo de híbridos blancos fue HB-83 y el de los amarillos fue HA-46, ambos de JC-TA-Guatemala.

Cuadro 3. Localidades en donde se evaluaron los ensayos de híbridos blancos del PCCMA. 1996.

\begin{tabular}{lllc}
\hline \multicolumn{1}{c}{ País } & \multicolumn{1}{c}{ Localidad } & Institución/Empresa & No. Ensayos \\
\hline Guatemala & San Jerónimo & JCTA & 1 \\
Guatemala & Jutiapa & JCTA & 1 \\
Guatemala & Tiquisate & C. BURKARD & 1 \\
Guatemala & Chiquimulilla & SEMJNAL & 1 \\
El Salvador & Sta. Cruz Porrillo & CENTA & 1 \\
Honduras & Playita & DICTA & 1 \\
Honduras & Omonita & DICTA & 1 \\
Honduras & Zamorano & EAP & 1 \\
Nicaragua & San Cristóbal & INTA & 1 \\
Nicaragua & Sta. Rosa & INTA & 1 \\
Panamá & El Ejido & IDJAP & 1 \\
México & Poza Rica & CJMMYT & 1 \\
& & & \\
& & Total de Ensayos & 12 \\
\hline
\end{tabular}

Cuadro 4. Localidades en donde se evaluaron los hfbridos amarillos del PCCMCA. 1996.

\begin{tabular}{lllc}
\hline \multicolumn{1}{c}{ País } & \multicolumn{1}{c}{ Localidad } & Institución/Empresa & No. Ensayos \\
\hline Guatemala & Cuyuta & JCTA & 1 \\
Guatemala & La Máquina & JCTA & 1 \\
Guatemala & Tiquisate & C. BURKARD & 1 \\
El Salvador & Sta Cruz Porcillo & CENTA & 1 \\
Honduras & Danlf & DICTA & 1 \\
Honduras & Zamorano & EAP & 1 \\
Nicaragua & Sant Cristóbal & INTA & 1 \\
Panamá & El Ejido & IDIAP & 1 \\
Panamá & Parita & IDJAP & 1 \\
Panamá & Rio Hato & IDIAP & 1 \\
México & Poza Rica & CJMMYT & 1 \\
Haití & Damién & CRDA & 1 \\
Rep. Dominic. & Baní & CESDA & 1 \\
Rep. Dominic. & San Cristóbal & CESDA & 1 \\
& & Total de Ensayos & 14 \\
\hline
\end{tabular}




\section{Variables medidas}

Se registraron las variables días a floración masculina y femenina, altura de planta y mazorca $(\mathrm{m})$, acame de raíz y tallo, enfermedades comunes, mazorcas descubiertas, aspecto de planta y mazorca, peso de campo de mazorca, porcentaje de humedad y ajuste de rendimiento al $15 \%$ de humedad.

\section{Análisis estadístico}

En los luoridos blancos el análisis de varianza por localidad de la variable rendimiento de grano se efectuó con el modelo de Látice y para los amarillos se utilizó el modelo de Bloques Completos al Azar.

$$
Y i j=U+T i+R j+E i j
$$

Donde:

$\mathrm{i}=1,2, \ldots, \mathrm{t} \quad \mathrm{t}=$ tratamiento

$\mathrm{j}=1,2, \ldots, \mathrm{r} \quad \mathrm{r}=$ repeticiones

Yij $=$ Valor del carácter estudiado de la parcelas con el i-ésimo tratamiento en la j-ésima repetición.

$\mathrm{U}=$ Media general del carácter

$\mathrm{Ti}=$ Efecto del i-ésimo tratamiento

$R j=$ Efectos de la j-ésima repetición

Eij $=$ Efectos aleatorio $\mathrm{s}$ asociados a la j-ésima observación

Se calculó la Diferencia Mínima Significativa (DMS) al 5\% para desglosar la variabilidad del rendimiento de grano (Separación de Medias).
Para medir la interacción genotipo-ambiente se efectuó el análisis de Efectos Principales Aditivos e Interacciones Multiplicativas (AMMI), utilizando el modelo descrito por Crossa; Gauch; Zobel (1990). Dicho modelo es el siguiente:

$$
\text { Yge }=\mu+\alpha g+\beta e+\underset{n=1}{N} \lambda \mathrm{n} \gamma g n \delta \text { en }+\rho g e
$$

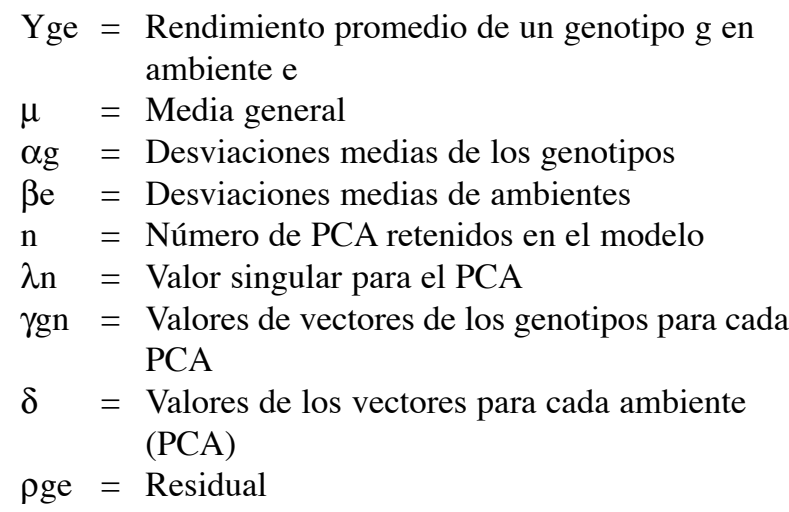

\section{RESULTADOS Y DISCUSIÓN}

\section{Ensayo de híbridos de grano blanco}

El Cuadro 5 presenta el análisis de varianza individual y combinado para rendimiento de grano de las 12 localidades. Hubo diferencias estadísticas entre las localidades y genotipos. La alta significancia de la interacción localidad por tratamiento (GXA) indica que los híbridos fueron influenciados de manera diferente en

Cuadro 5. Análisis de varianza por localidad de la variable rendimiento de grano blanco del PCCMCA 1996

\begin{tabular}{rlrcc}
\hline & & \multicolumn{3}{c}{ Media } \\
\cline { 3 - 5 } No. & & C.M. & t/ha & C.V (\%) \\
\hline & & & & \\
1 & San Jerónimo (ICTA) & $2,926 * *$ & 8,419 & 6,10 \\
2 & Jutiapa (ICTA) & $0,848^{* *}$ & 6,778 & 4,70 \\
3 & Tiquisate (C. Burkard) & $1,815 * *$ & 6,412 & 6,50 \\
4 & Chiquimulilla (Seminal) & $2,547 * *$ & 4,412 & 15,27 \\
5 & Sta. Cruz Porrillo (CENTA) & $1,475 * *$ & 4,511 & 13,50 \\
6 & Playita (DICTA) & $4,216 * *$ & 4,364 & 10,48 \\
7 & Omonita (DICTA) & $0,695 * *$ & 6,020 & 7,19 \\
8 & Zamorano (EAP) & $3,799 * *$ & 6,054 & 18,92 \\
9 & San Cristóbal (INTA) & $3,258 * *$ & 6,714 & 10,71 \\
10 & Sta. Rosa (INTA) & $1,117 * *$ & 6,184 & 9,54 \\
11 & El Ejido (IDIAP) & $2,381 * *$ & 5,011 & 10,64 \\
12 & Poza Rica (CIMMYT) & $6,710 * *$ & 5,170 & 14,29 \\
& Combinado & $15,145 * *$ & 5,815 & 3,00 \\
& Interacción LocXTrat & $1,513 * *$ & & \\
\hline
\end{tabular}

$\mathrm{CM}=$ Cuadrado medio de tratamientos

$\mathrm{CV}=$ Coeficiente de variación

$* *=$ Altamente significativo $(\mathrm{a}=0,01)$ 
los distintos ambientes evaluados. El coeficiente de variación $(\mathrm{CV})$ varió entre 3,0 y 18,9\%, lo cual se. con-sidera adecuado y representan la confiabilidad en la conducción de los experimentos. Todas las localidades mostraron rendimientos promedios por encima de 4,0 t/ha, y la localidad de San Jerónimo mostró los rendimientos más altos $(8,42 \mathrm{t} / \mathrm{ha})$.

En el análisis AMMI se observó alta significancia estadística para todas las fuentes de variación en el estudio. Los resultados muestran que el PCA1 captó el $31,32 \%$ de la suma de cuadrados de la interacci6n localidad x híbrido con el 12,23\% de los grados de libertad.

De los 30 híbridos blancos evaluados en 1996, sólo seis superaron o igualaron en rendimiento al testigo HB-83 con puntuaciones AMMI cercanas a cero (Cuadro 6, Figura 1), estos fueron A-7573, H-53, HN-951, A-7530, CB-XHS-7GM1 y CML-9xCML-47. De estos, tres pertenecen a los programas nacionales y tres a las compañías privadas de semilla (Cristiani-Bur-kard y Asgrow). Otros materiales con buen potencial de rendimiento pero interacción significativa con el am-biente (puntuaciones AMMI lejanas a cero) fueron A-XM7598, CB-XHS-5GM2, HE-59, MBHB-51 Y CML247x CML-254 (Cuadro 6).

\section{Ensayo de híbridos de grano amarillo}

El análisis de varianza por localidad y combinado de rendimiento se presenta en el Cuadro 7, en todas las localidades la respuesta fue altamente significativa, excepto en Haití, donde fue significativa al $0,05 \%$ de probabilidad, la localidad que reportó el más alto promedio de rendimiento fue Danlí, Honduras con 7,068 t/ha. Los coeficientes de variación presentan valores aceptables, únicamente en Haití presenta un CV alto $(35,66 \%)$.

El Cuadro 8 presenta el análisis AMMI para rendimiento de los 20 híbridos amarillos a través de 14 localidades. Los resultados mostraron alta significancia estadística para todas la fuentes de variación. El primer eje de interacción PCA 1 captó el 27,04\% de la suma de cuadrados de la interacción localidad x híbrido con $12,55 \%$ de los grados de libertad. Las localidades mostraron rendimientos promedios de 3,7 a 7,0 t/ha, siendo Danlí la de mayor rendimiento promedio (Cuadro 9).

De los 20 híbridos amarillos evaluados, sólo tres superaron al testigo HA-46 (en 8,3; 4,0 y 6,2\%, respectivamente) con puntuaciones AMMI cercanas a cero, estos fueron CB-XHS-8GM3, HS-6 y DK-888A, todos pertenecientes a las compañías privadas de semilla (Cuadro 9, Figura 2). El híbrido de mayor rendimiento fue CML-287xCL-331 con rendimiento de 6,17 t/ha superó al testigo HA-46 en $14,02 \%$, pero con puntuación AMMI de 0,416, mostrando una interacción significativa con el ambiente.

\section{CONCLUSIONES}

El comportamiento de los híbridos blancos y amarillos fue similar, con una media de rendimiento de 5,82 t/ha para los híbridos de grano blanco y de 5,12 t/ha para los amarillos. Sin embargo, se identificaron más genotipos en los híbridos blancos que superaron al testigo que en los materiales amarillos.

Varios híbridos de los Programas Nacionales y las Empresas Privadas fueron superiores a los testigos, en más de $15 \%$, por lo que se deduce progreso significativo en la generación de germoplasma.

El comportamiento y potencial productivo de algunos híbridos fue afectado por la mala calidad de la semilla (baja germinación y vigor) lo que tuvo como consecuencia la pérdida de densidad de población, además en ciertas localidades el cultivo fue afectado por altas precipitaciones.

Con el objetivo de que los agricultores dispongan de genotipos con alto potencial de rendimiento, se recomienda impulsar los mejores híbridos a nivel de transferencia.

\section{RECONOCIMIENTOS}

La presente investigación es producto de un trabajo cooperativo en el cual han participado entidades e instituciones de Centro América, Panamá, El Caribe y México, en donde los trabajos se han llevado a cabo con mucho interés y profesionalismo.

El Salvador: Adán Aguiluz, Didencio Guerra, Fausto Zavala (CENTA).

Guatemala: Carlos Pérez, José Luis Quemé, Mario Roberto Fuentes, José Luis Zea, Luis Larios (ICTA). Antonio Cristiani B., (CRISTIANI BURKARD), René Velásquez (SEMINAL).

Honduras: Luis Brizuela, Danilo Escoto, Ouit Zapata, Santos R. Calix (DICTA), Francisco Gómez (El Zamorano). Alejandro Palma (Dekalb). Carlos H. Merlo (Cargil).

Nicaragua: Róger Urbina, Marcos Mendoza (INTA). 
Cuadro 6. Puntuaciones para híbridos de grano blanco y ambientes del primer eje de interacción (AMMI), PCCMCA 1996.

\begin{tabular}{|c|c|c|c|c|c|c|}
\hline No & Híbridos & $\begin{array}{c}\text { Rend } \\
\text { t/ha }\end{array}$ & $\begin{array}{l}\text { Punt } \\
\text { AMMI }\end{array}$ & Ambientes & $\begin{array}{c}\text { Rend } \\
\text { t/ha }\end{array}$ & $\begin{array}{l}\text { Punt } \\
\text { AMMI }\end{array}$ \\
\hline 13 & C-381 & 5,359 & 1,240 & 8 Zamorano (EAP) & 6,054 & 0,903 \\
\hline 2 & HB-85 & 4,867 & 1,110 & 2 Jutiapa (ICTA) & 6,777 & 0,668 \\
\hline 23 & XM-7595 & 5,679 & 0,659 & 1 San Jerónimo (ICTA) & 8,419 & 0,430 \\
\hline 19 & A-775 & 5,597 & 0,610 & 3 Tiquisate (C. Burkard) & 6,412 & 0,336 \\
\hline 16 & CB-XHS-5GM1 & 5,512 & 0,586 & 10 Sta. Rosa (INTA) & 6,185 & 0,256 \\
\hline 1 & HB-83 & 5,942 & 0,494 & 11 El Ejido (IDIAP) & 5,011 & 0,230 \\
\hline 25 & MAX-329 & 5,853 & 0,384 & 7 Omonita (DICTA) & 6,020 & 0,225 \\
\hline 3 & HE-9101 & 5,172 & 0,348 & 4 Seminal & 4,143 & 0,061 \\
\hline 11 & $\mathrm{C}-220$ & 5,679 & 0,268 & 5 Sta.Cruz Ponillo (CENTA) & 4,511 & $-0,093$ \\
\hline 22 & A-7573 & 6,998 & 0,151 & 9 San Cristóbal (INTA) & 6,714 & $-0,350$ \\
\hline 9 & H-53 & 5,949 & $-0,007$ & 6 Playita (DICTA) & 4,364 & $-0,416$ \\
\hline 24 & MAX-325 & 4,942 & $-0,009$ & 12 Poza Rica (CIMMYT) & 5,171 & $-2,250$ \\
\hline 6 & HN-951 & 6,010 & $-0,073$ & & & \\
\hline 5 & HN-953 & 5,619 & $-0,141$ & & & \\
\hline 28 & HR-93 & 4,940 & $-0,180$ & & & \\
\hline 27 & HR-93M & 5,356 & $-0,197$ & & & \\
\hline 20 & A-7530 & 6,489 & $-0,206$ & & & \\
\hline 18 & CB-XHS-7GM1 & 6,426 & $-0,223$ & & & \\
\hline 4 & $\mathrm{HN}-955 \mathrm{~A}$ & 5,820 & $-0,235$ & & & \\
\hline 15 & DK-880 & 5,608 & $-0,262$ & & & \\
\hline 30 & CML-9xCML-47 & 7,056 & $-0,269$ & & & \\
\hline 12 & C-526 & 4,650 & $-0,273$ & & & \\
\hline 8 & MBHB-52 & 5,028 & $-0,279$ & & & \\
\hline 21 & A-XM-7598 & 6,349 & $-0,294$ & & & \\
\hline 26 & MAX-327 & 5,650 & $-0,300$ & & & \\
\hline 17 & CB-XHS-5GM2 & 6,304 & $-0,387$ & & & \\
\hline 10 & HE-59 & 6,812 & $-0,466$ & & & \\
\hline 14 & AGX-5488 & 5,744 & $-0,520$ & & & \\
\hline 7 & MBHB-51 & 6,120 & $-0,602$ & & & \\
\hline \multirow[t]{2}{*}{29} & CML-247xCML-254 & 6,923 & $-0,926$ & & & \\
\hline & Media General & 5,817 & & & & \\
\hline
\end{tabular}

Panamá $\quad$ Alfonso Alvarado, Daniel Pérez, Timoteo Ponce (IDIAP).

Cuba: $\quad$ Cecilio Torres (IIHLL).

Haití: $\quad$ Juan René Bossa (CRDA).

R. Dominicana Richard Ortiz, Félix Navarro, (CESDA).

México: Humberto Gutiérrez (ASGROW), Federico Poey (AGRIDEC), Sunrider Vasal (CIMMYT).

CIMMYT: Jorge Bolaños, William Quemé, Miriam Hernández, Elio Durón (PRM).
Los coordinadores de los Programas Nacionales de Maíz (CRP) agradecen a las compañías de semillas que operan en la región, por el apoyo económico brindado para la realización de esta investigación.

CRISTIANI BURKARD
AGRIDEC
ASGROW
AGROCERES
SEMINAL
CARGIL
DEKALB

CRISTIANI BURKARD

ASGROW

DEKALB 

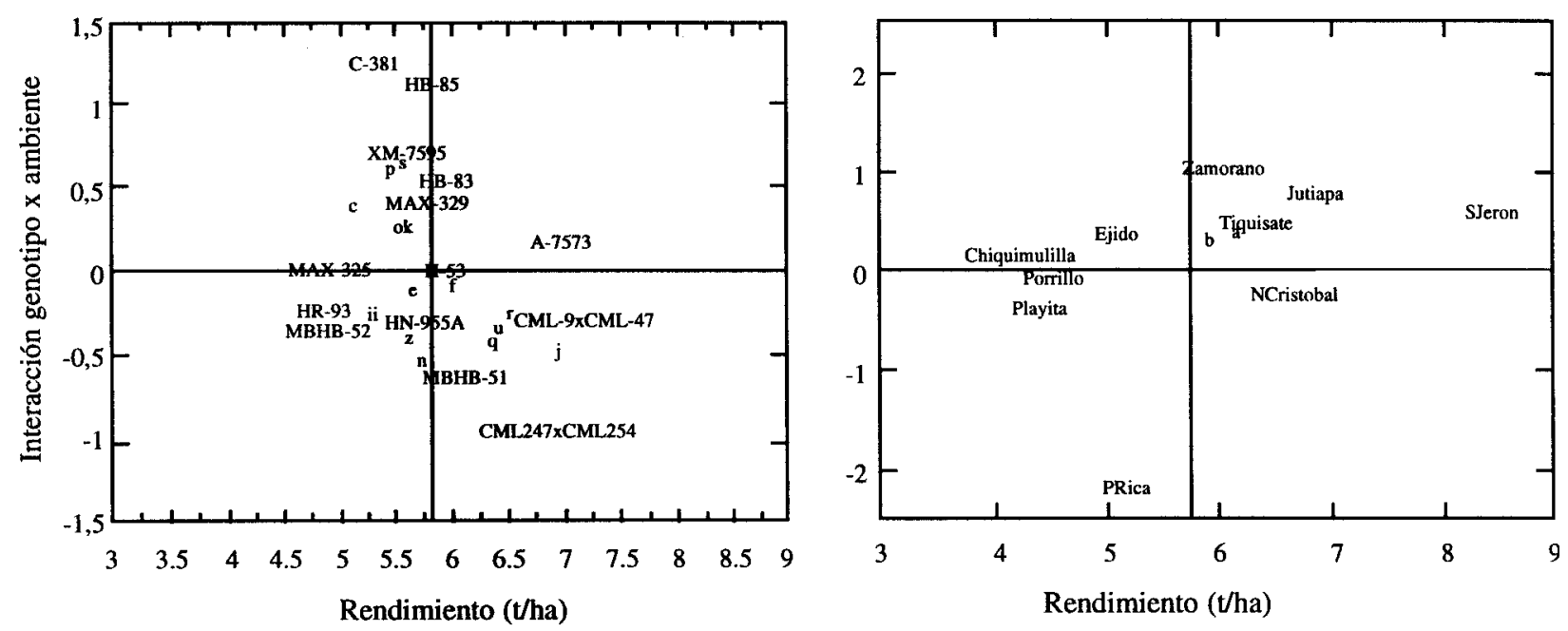
e HE-9101
n AGX-5488
t A-7560
a Sta. Rosa Nic.
e $\mathrm{HN}-953$
o DK-880
u A-XM-7598
b Omonita
f HN-95 1
P CB-XHS-5GMI
z MAX-327
j HE-59
q CB-XHS-5GM2
ii HR-93M
k C-220
r CB-XHS-7GMI
i C-526
s $\mathrm{A}-775$

Figura 1. Medias de rendimiento y puntuaciones del primer eje del componente principal de 30 híbridos de grano blanco y 12 ambientes. PCCMCA, 1996.

Cuadro 7. Análisis de varianza por localidad de la variable rendimiento híbridos de grano amarillo, PCCMCA 1996.

\begin{tabular}{rlccr}
\hline No. & \multicolumn{1}{c}{ Localidad } & CM & Media t/ha & CV(\%) \\
\hline 1 & Cuyuta (ICTA) & $2,326 * *$ & 3,758 & 13,44 \\
2 & La Máquina (ICTA) & $2,548 * *$ & 4,379 & 7,12 \\
3 & Tiquisate (c. BURKARD) & $1,237 * *$ & 6,365 & 5,81 \\
4 & Sta. Cruz Porrillo (CENTA) & $1,282 * *$ & 3,874 & 15,65 \\
5 & Danlí (DICTA) & $1,302 * *$ & 7,068 & 3,99 \\
6 & Zamorano (EAP) & $2,854 * *$ & 6,063 & 14,02 \\
7 & San Cristóbal (INTA) & $1,737 * *$ & 6,438 & 8,01 \\
8 & El Ejido (IDIAP) & $1,334 * *$ & 4,960 & 7,62 \\
9 & Parita (IDIAP) & $1,478 * *$ & 4,422 & 9,59 \\
10 & Rio Hato (IDIAP) & $1,398 * *$ & 5,378 & 6,78 \\
11 & Poza Rica (CIMMYT) & $5,617 * *$ & 3,812 & 11,57 \\
12 & Haití (CRDA) & $3,348 *$ & 3,771 & 35,66 \\
13 & Baní (CESDA) & $3,132 * *$ & 5,190 & 16,70 \\
14 & San Cristóbal (CESDA) & $1,237 * *$ & 6,365 & 5,81 \\
& & & & \\
& Combinado & $11,840 * *$ & 5,116 & 3,49 \\
& Loc x Trat & $1,556 * *$ & & \\
\hline
\end{tabular}

$\mathrm{CM}=$ Cuadrado medio de tratamientos

$\mathrm{CV}=$ Coeficiente de variación

NS $=$ No significativo

$* *=$ Altamente significativo $(\mathrm{a}=0,01)$

$*$ = Significativo $(\mathrm{a}=0,05)$ 
Cuadro 8. Análisis AMMI para rendimiento de 20 hí'bridos de grano amarillo evaluados en 14 localidades PCCMCA 1996.

\begin{tabular}{lrrrl}
\hline \multicolumn{1}{c}{ Fuente } & G.L & \multicolumn{1}{c}{ S.C } & C.M. & Probabilidad \\
\hline Localidades & 13 & 1024,522 & 78,809 & $0,0000^{* * * *}$ \\
Híbridos & 19 & 224,712 & 11,827 & $0,0000^{* * * *}$ \\
Localidad x Hdo & 247 & 386,163 & 1,563 & $0,0000^{* * * *}$ \\
PCAl & 31 & 104,409 & 3,368 & $0,0000^{* * * *}$ \\
PCA2 & 29 & 83,607 & 2,883 & $0,000^{* * * *}$ \\
Error & 532 & 380,879 & 0,716 & \\
Total & 839 & $2.090,850$ & 2,492 & \\
\hline
\end{tabular}

Cuadro 9. Puntuaciones para híbridos de color amarillo y ambientes del primer eje de interacción (AMMI), PCCMCA 1996.

\begin{tabular}{|c|c|c|c|c|c|c|c|}
\hline No. & Híbrido & $\begin{array}{c}\text { Rend } \\
\text { t/ha }\end{array}$ & $\begin{array}{l}\text { Punt } \\
\text { AMMI }\end{array}$ & & Ambientes & $\begin{array}{c}\text { Rend } \\
\text { t/ha }\end{array}$ & $\begin{array}{l}\text { Punt } \\
\text { AMMI }\end{array}$ \\
\hline 2 & MBHA-53 & 4,841 & 1,017 & 11 & Poza Rica (CIMMYT) & 3,812 & 1,324 \\
\hline 1 & RD-662 & 4,514 & 0,952 & 12 & Haití (CRDA) & 3,771 & 0,974 \\
\hline 16 & CB-XHS-8GM2 & 5,836 & 0,793 & 2 & La Máquina (ICTA) & 4,379 & 0,342 \\
\hline 19 & CML-287xCL-331 & 6,171 & 0,416 & 3 & Tiquisate (C. BURKARD) & 6,365 & 0,204 \\
\hline 9 & HE-9126 & 5,028 & 0,346 & 1 & Cuyuta (ICTA) & 3,758 & 0,188 \\
\hline 3 & MBHA-54 & 4,627 & 0,308 & 7 & San Cristóbal (INTA) & 6,438 & 0,172 \\
\hline 17 & CB-XHS-8GM3 & 5,859 & 0,197 & 14 & San Cristóbal (CESDA) & 6,148 & 0,154 \\
\hline 5 & C-701 & 4,686 & 0,055 & 9 & Parita (IDIAP) & 4,422 & 0,013 \\
\hline 10 & H-104 & 5,389 & $-0,016$ & 4 & Sta. Cruz Porrillo (CENTA) & 3,874 & $-0,128$ \\
\hline 7 & HA-46 & 5,412 & $-0,035$ & 8 & El Ejido (IDIAP) & 4,961 & $-0,173$ \\
\hline 8 & HE-9122 & 5,208 & $-0,075$ & 10 & Rio Hato (IDIAP) & 5,379 & $-0,350$ \\
\hline 11 & P-8916 & 4,988 & $-0,077$ & 5 & Danlí (DICTA) & 7,067 & $-0,452$ \\
\hline 18 & HS-6 & 5,630 & $-0,077$ & 13 & Baní (CESDA) & 5,190 & $-1,108$ \\
\hline 13 & DK-888A & 5,748 & $-0,117$ & 6 & Zamorano (EAP) & 6,063 & $-1,160$ \\
\hline 4 & C-805 & 4,639 & $-0,358$ & & & & \\
\hline 12 & P-8812 & 4,721 & $-0,424$ & & & & \\
\hline 15 & AG-9014 & 4,856 & $-0,483$ & & & & \\
\hline 20 & ESA4xESA2 & 4,535 & $-0,546$ & & & & \\
\hline 14 & AG-510 & 5,311 & $-0,575$ & & & & \\
\hline \multirow[t]{2}{*}{6} & C-425 & 4,325 & $-1,302$ & & & & \\
\hline & Media General & 5,116 & & & & & \\
\hline
\end{tabular}




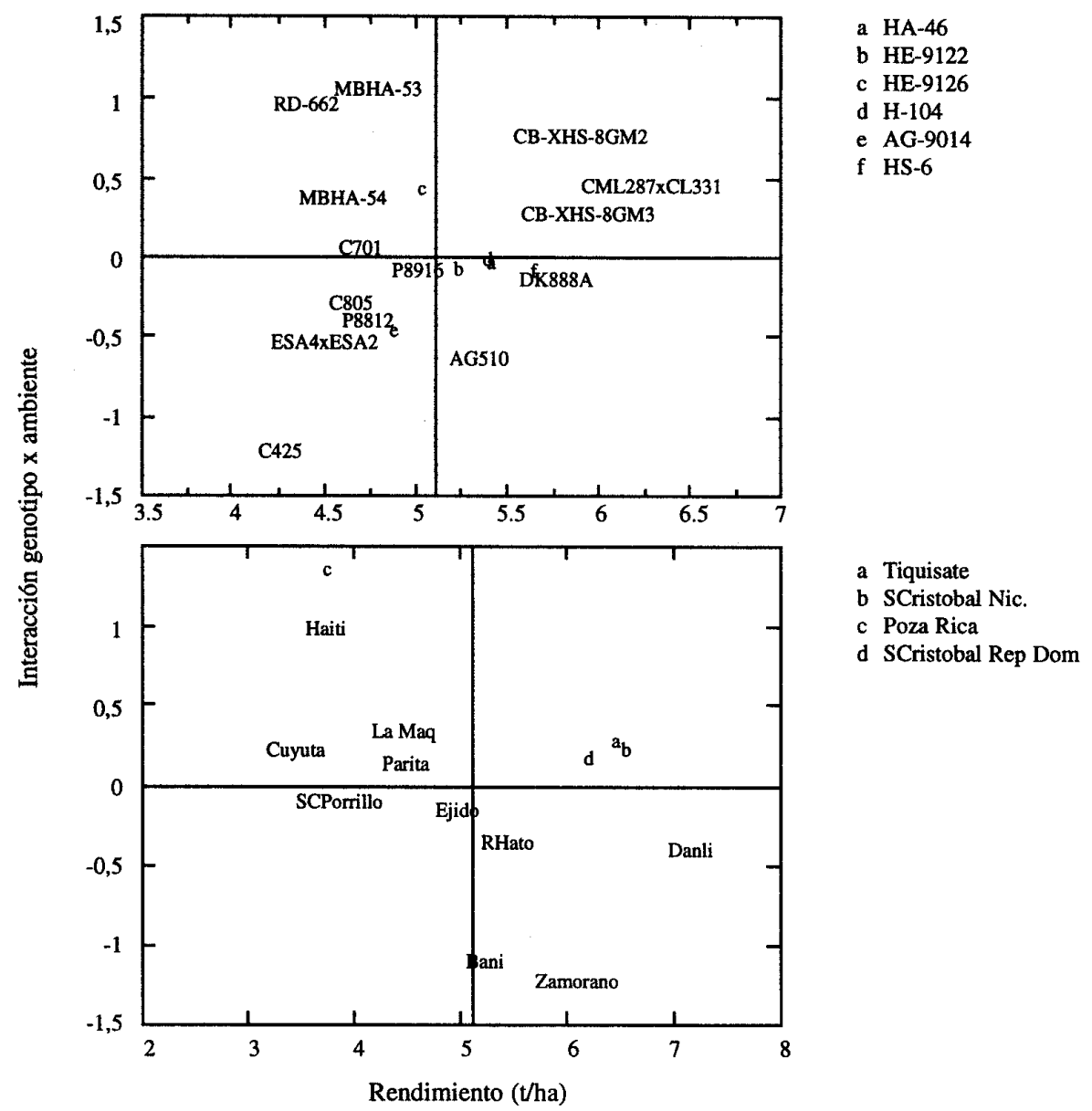

Figura 2. Medias de rendimiento y puntuaciones del primer eje del componente principal de 20 híbridos de grano amarillo y 14 ambientes. PCCMCA, 1996.

\section{LITERATURA CITADA}

ALVARADO A.; CAMARGO I. B.; GORDÓN R, 1994. Evaluación de híbrido s de maíz (Zea mays) de grano blanco y amarillo, en ambientes de Centroamérica, Panamá y El Caribe. Informe PCCMCA 1994. Panamá, 37 p.

BRIZUELA L. 1995. Evaluación de híbridos de maíz (Zea mays L.) de grano blanco y amarillo en ambientes de Centroamérica, Panamá y El Caribe. Informe PCCMCA 1996, El Salvador.

CORDOVA, H.S. 1989. Evaluación de 36 cultivares de maíz en 20 ambientes de Centro América, Panamá y El Caribe, PCCMCA 1988. Presentado en la XXXV Reunión Anual del PCCMCA, San Pedro Sula, Honduras.

CROSSA, J.; GAUCH H.G. Jr.; ZOBEL R,W. 1988. Estimación estadística predictiva de rendimiento en ensayos de variedades. In Simposio "Modelos de estabilidad para evaluar la adaptación de cultivares. XXXIV Reunión Anual del PCCMCA, San José, Costa Rica.

QUEME, J.L.; FUENTES M. R. 1992. Evaluación de híbridos de maíz (Zea mays L.) de grano blanco y amarillo, en diferentes ambientes de México, Centro América, El Caribe y Venezuela. Informe PCCMCA 1991 PRM-ICTA, Guatemala, C.A. p. 61.

URBINA, R, 1992. Evaluación de híbridos de maíz (Zea mays L.) de grano blanco y amarillo en ambientes de Centro América, Panamá, El Caribe y México. Informe PCCMCA 1993. Guatemala CA p. 60.

PIXLEY L. 1993. Evaluación de híbridos de maíz (Zea mays L.) de grano blanco y amarillo en ambientes de Centro América, Panamá, y El Caribe. Informe PCCMCA 1994 Tegucigalpa Honduras. 